\title{
A novel gross deletion and breakpoint junction sequence analysis of $A T P 7 B$ in a Chinese family with Wilson disease using next-generation sequencing and Sanger sequencing
}

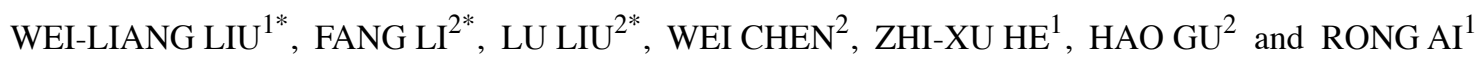 \\ Departments of ${ }^{1}$ Pediatrics and ${ }^{2}$ Ophthalmology, Affiliated Hospital of Guizhou Medical University, \\ Guiyang, Guizhou 550004, P.R. China
}

Received April 25, 2019; Accepted October 15, 2019

DOI: $10.3892 / \mathrm{mmr} .2019 .10830$

\begin{abstract}
Wilson disease (WD) is a rare autosomal recessive genetic disorder that causes abnormal copper metabolism, resulting in pathological accumulation of copper in the liver, brain and other organs. Mutations in the ATPase copper transporter 7B (ATP7B) gene, which encodes a membrane P-type adenosine triphosphatase, have been identified as being responsible for WD. The present study analyzed clinical data and collected DNA samples from a pediatric patient with WD and her healthy parents. Mutation screening for $A T P 7 B$ was performed using direct sequencing, multiplex ligation-dependent probe amplification(MLPA), next-generation sequencing (NGS) and Sanger sequencing of the breakpoint junction sequence. The patient (age, 2.7 years) presented with early-onset hepatic disease. The present study identified compound heterozygous mutations of $A T P 7 B$, including a heterozygous mutation (p.Arg1,041Trp) and a novel heterozygous gross deletion of a 57,771 bp fragment (chr13: 52490972-52548742) (GRCh37) from partial exon2- exon21 to external $A T P 7 B$ sequence (15.833bp) in the patient. Analysis of the family members of the patient showed that the missense mutation and the gross deletion mutation were inherited from her mother and father, respectively. Microhomology and inverted repeat sequences, which may mediate the deletion mutation, were identified through sequence analysis on both sides of the breakpoints of this deletion. The present study provided additional information on the genotypic spectrum of the $A T P 7 B$ gene, particularly with regard to early onset hepatic disease,
\end{abstract}

Correspondence to: Dr Wei-Liang Liu or Dr Zhi-Xu He, Department of Pediatrics, Affiliated Hospital of Guizhou Medical University, 28 Guiyi Street, Guiyang, Guizhou 550004, P.R. China E-mail: liuweiliang205@aliyun.com

E-mail:hzx@gmc.edu.cn

${ }^{*}$ Contributed equally

Key words: Wilson disease, child, ATPase copper transporter B, next-generation sequencing, mutation as observed in the present patient with WD. The identification of the precise breakpoint junction sequence warrants further investigation of DNA break and recombination mechanisms. In detecting precise deletions, the NGS associated with Sanger sequencing of breakpoint junction sequence have been found to have more advantages than MLPA.

\section{Introduction}

Wilson disease (WD; OMIM 27790) is a rare autosomal recessive disorder affecting copper transport, characterized by decreased biliary copper excretion and reduced copper incorporation into ceruloplasmin (1). The worldwide incidence is $\sim 30$ per million individuals, with a gene frequency of $0.56 \%$ and a carrier frequency of $1 / 90$ (2). The main clinical features are hepatic, neurologic and psychiatric disorders and, Kayser-Fleischer (KF) rings (3). Defects the ATPase copper transporter 7B $(A T P 7 B)$ gene, which encodes for a membrane P-type ATPase, at $13 \mathrm{q} 14.3$ have been identified to be responsible for WD (4). The gene spans $\sim 80 \mathrm{~kb}$ and consists of 21 exons. $A T P 7 B$ encodes a protein with 1,465 amino acid that consists of six copper binding domains, eight transmembrane domains and one ATP loop that transports copper into bile (5). The present study identified a heterozygous missense mutation, which, to the best of our knowledge, has not been previously identified in the Chinese population. In addition, the present analysis identified a novel heterozygous gross deletion mutation in the $A T P 7 B$ locus in a Chinese pediatric patient with WD. Additionally, the sequence of the precise breakpoint junction was validated. Analysis of the family members showed that the missense mutation and the novel gross deletion mutation were inherited from the mother and father, respectively.

\section{Subjects and methods}

Subjects. The proband was hospitalized at the department of Pediatrics of the Affiliated Hospital of Guizhou Medical University (Guiyang, China) due to anorexia in March 2015. The present study was approved by the Ethics Committees of Affiliated Hospital of Guizhou Medical University (approval no. 201011). Written informed consent was obtained from 
the parents of the patient. Peripheral venous blood samples ( $3 \mathrm{ml})$, were collected from each subject. In addition, the following parameters of hospital routine examination were analyzed: Alanine transaminase (ALT), aspartate transaminase (AST), ceruloplasmin (CER), creatine kinase, myocardial band isoenzyme of creatine kinase, lactate dehydrogenase, ammonia, total bilirubin, lactic acid, $\alpha$ fetal protein, blood urea nitrogen, blood routine, urine routine, antibody to hepatitis viruses A-E, Epstein-Barr virus IgM, toxoplasmosis, rubella, cytomegalovirus and herpes simplex virus screening, and autoimmune hepatitis antibodies and antinuclear antibody spectrum. Brain MRI and abdominal CT were performed on the patient. In total, three people were enrolled; a female patient with WD (age, 2.7 years) and her father and mother (aged 29 and 28 years, respectively).

Genetic analysis. Blood samples $(3 \mathrm{ml})$ were collected from every family member after informed consent was obtained, including from the parents of the patient. Genomic DNA was then isolated from peripheral blood lymphocytes using the Tianamp Genomic DNA kit (DP304; Tiangen Biotech Co., Ltd.) according to the manufacturer's recommendations.

Sanger-method sequencing. All of the coding exons and exon-intron boundaries of $A T P 7 B$ were amplified by PCR using the same primers as previously described amplification conditions (6). The PCR products were sequenced using ABI PRISM 3730XL DNA automated sequencer (Applied Biosystems; Thermo Fisher Scientific, Inc.)

Multiplex ligation-dependent probe amplification (MLPA). The DNA in which two mutations in the $A T P 7 B$ gene could not be identified by sequencing was subsequently tested for partial exon deletions/duplications using the ATP7B MLPA assay (SALSA MLPA kit Wilson disease; MRC-Holland BV) including primers and enzymes according to the manufacturer's protocol. The thermocycling conditions included initial denaturation at $98^{\circ} \mathrm{C}$ for $5 \mathrm{~min}$, pause at $25^{\circ} \mathrm{C}$, hybridisation reaction at $95^{\circ} \mathrm{C}$ for $1 \mathrm{~min}, 60^{\circ} \mathrm{C}$ for $16 \mathrm{~h}$, ligation reaction at $54^{\circ} \mathrm{C}$ for $15 \mathrm{~min}, 98^{\circ} \mathrm{C}$ for $5 \mathrm{~min}$, pause at $20^{\circ} \mathrm{C}$, followed by 35 cycles of $95^{\circ} \mathrm{C}$ for $30 \mathrm{sec}, 60^{\circ} \mathrm{C}$ for $30 \mathrm{sec}, 72^{\circ} \mathrm{C}$ for $60 \mathrm{sec}$, and an extension at $72^{\circ} \mathrm{C}$ for $20 \mathrm{~min}$.

Next-generation sequencing (NGS). The DNA in which precise heterozygous deletions in the $A T P 7 B$ gene could not be identified by MLPA was subsequently tested with NGS. The genomic DNA sample was sheared by sonication. A xGen Exome Research Panel v1.0 kit (Integrated DNA Technologies, Inc.) was used to capture exons; the full length of all exons was of $35.58 \mathrm{Mb}$. The samples were then sequenced on an Illumina Hiseq2500 System (Illumina, Inc.). The sequencing reads were aligned to the human reference genome (GRCH37/hg19) using Burrows-Wheeler Aligner (ver. 0.7.12, http://bio-bwa. sourceforge.net/). Single nucleotide variations, insertions and deletions were analyzed with Samtools and indel software (https://sourceforge.net/projects/samtools/files/). Selected variants were checked in relevant variant frequency databases( (Exome sequencing project (http://evs.gs.washington. edu/EVS/), dbSNP (http://www.ncbi.nlm.nih.gov/SNP/),
Table I. Clinical data of the proband.

\begin{tabular}{lcc}
\hline Clinical data & Patient & Normal range \\
\hline ALT & $126.4 \mathrm{U} / 1$ & $7-40 \mathrm{U} / 1$ \\
AST & $86.5 \mathrm{U} / 1$ & $13-35 \mathrm{U} / 1$ \\
CER & $9.77 \mathrm{mg} / \mathrm{dl}$ & $22-58 \mathrm{mg} / \mathrm{dl}$ \\
CK & $179.2 \mathrm{U} / 1$ & $26-140 \mathrm{U} / 1$ \\
LDH & $301.1 \mathrm{U} / 1$ & $109-245 \mathrm{U} / 1$ \\
AMM & $80 \mu \mathrm{mol} / 1$ & $18-72 \mu \mathrm{mol} / 1$ \\
CKMB & $22.49 \mathrm{U} / 1$ & $0.0-25 \mathrm{U} / 1$ \\
TBIL & $5.96 \mu \mathrm{mol} / 1$ & $3.4-17 \mu \mathrm{mol} / 1$ \\
LACT & $1.93 \mathrm{mmol} / 1$ & $0.6-2.2 \mathrm{mmol} / 1$ \\
AFP & $3.1 \mathrm{ng} / \mathrm{ml}$ & $0.0-8.1 \mathrm{ng} / \mathrm{ml}$ \\
BUN & $3.61 \mathrm{mmol} / 1$ & $1.78-7.14 \mathrm{mmol} / 1$ \\
Blood routine & - & \\
Urine routine & - & \\
Antibody to hepatitis & - & Normal \\
viruses A-E & & \\
Epstein-Barr virus IgM & - & \\
TORCH & - & \\
Autoimmune hepatitis & - & \\
antibodies & Negative & \\
ANA antibody spectrum & Normal & \\
Brain MRI & Abnormal & \\
Abdominal CT & - & \\
\hline
\end{tabular}

ALT, alanine transaminase; AST, aspartate transaminase; CER, ceruloplasmin; CK, creatine kinase; LDH, lactate dehydrogenase; AMM, ammonia; CKMB, myocardial band isoenzyme of creatine kinase; TBIL, total bilirubin; LACT, lactic acid; AFP, $\alpha$ fetal protein; BUN, blood urea nitrogen; TORCH, toxoplasmosis, rubella, cytomegalovirus, herpes simplex virus; ANA antibody spectrum, antinuclear antibody spectrum

1000Genomes (http://www.1000genomes.org/), ClinVar (https://www.ncbi.nlm.nih.gov/clinvar/) and Human gene mutation database (http://www.hgmd.cf.ac.uk/ac/index.php)). Pathogenicity prediction tools (Polyphen 2 (http://genetics. bwh.harvard.edu/pph2/); SIFT (http://sift.jcvi.org/); Mutation Taster (http://www.mutationtaster.org/)) were additionally used.

Junction sequence confirmed by Sanger-method sequencing. The possible region deleted in $A T P 7 B$ was detected by NGS. The suspected breakpoint junction sequence was amplified using PCR. Specific primers were designed on both sides of the breakpoints. The following primers were used: F: 5'-GCC AGAGAAGCTGGGATGTT-3' and R: 5'-GACTGACCC AGCCCTCTTTC-3'. DNA polymerase was provided by the Tianamp Genomic DNA kit (ET105; Tiangen Biotech Co., Ltd.).

The thermocycling conditions were: Initial denaturation at $95^{\circ} \mathrm{C}$ for $5 \mathrm{~min}$, followed by 30 cycles of $95^{\circ} \mathrm{C}$ for $30 \mathrm{sec}$, $62^{\circ} \mathrm{C}$ for $30 \mathrm{sec}, 72^{\circ} \mathrm{C}$ for $30 \mathrm{sec}$, and a final extension at $72^{\circ} \mathrm{C}$ for $10 \mathrm{~min}$. The amplification product of the gel extraction purification was sequenced using ABI PRISM 3730XL DNA 
A

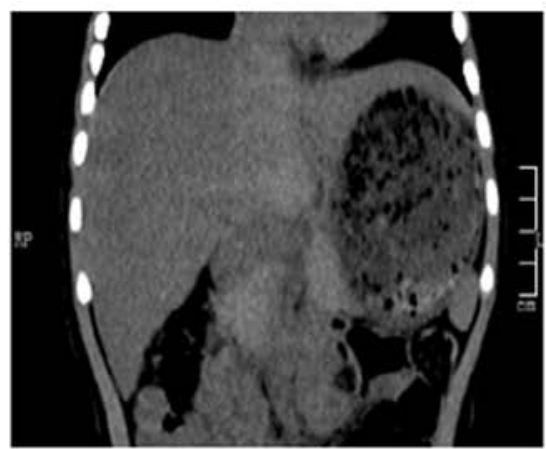

C

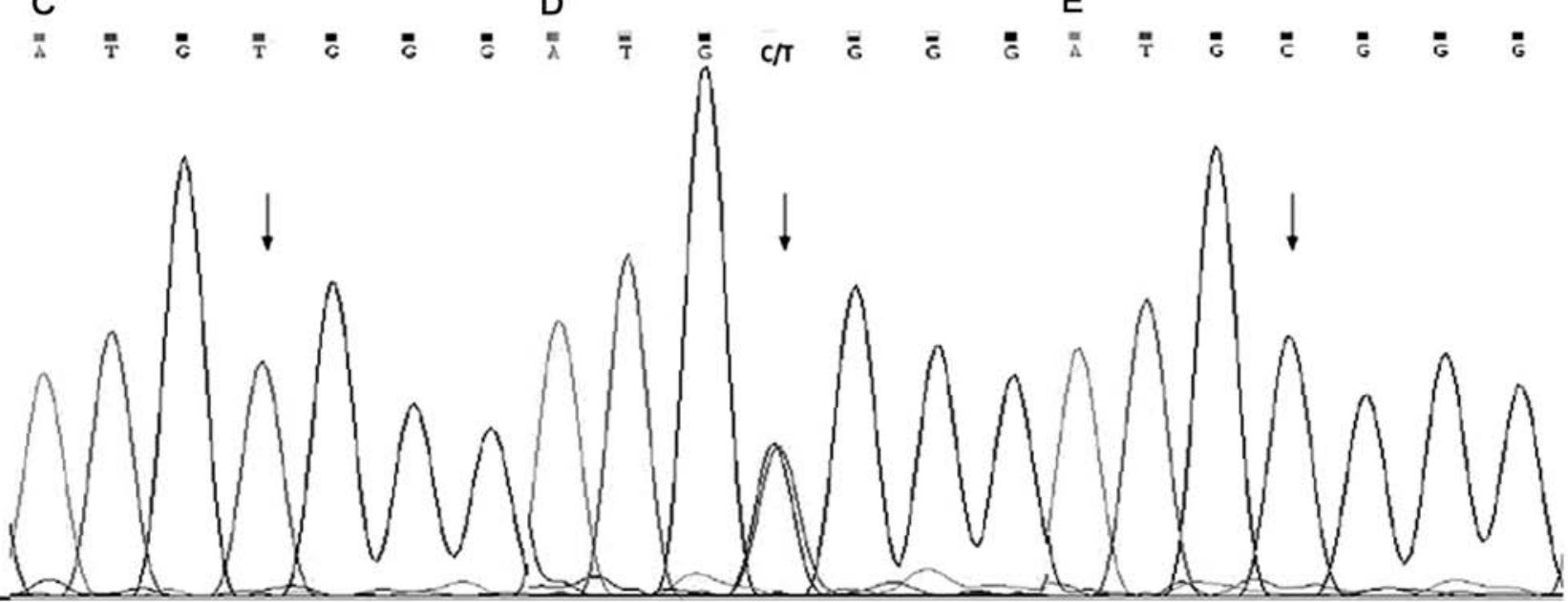

B

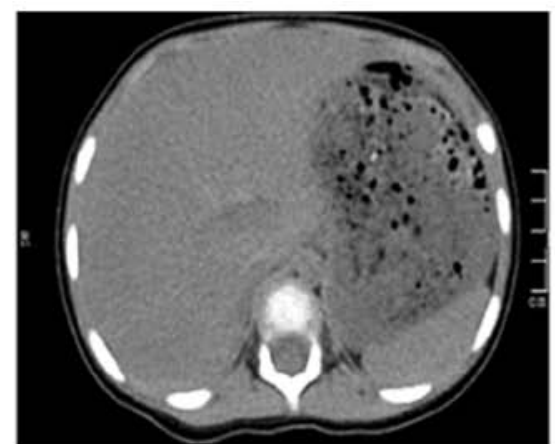

$\mathrm{E}$

Figure 1. CT scans of the patient and a heterozygous missense mutation analysis in the family. (A) Coronal CT of the abdomen of the patient. (B) Axial CT of the abdomen of the patient. Abdominal CT of the patient with WD showed an increased volume and decreased density of the liver. (C) Direct sequencing showed a heterozygous mutation (c.3121C >T; p.Arg1,041Trp) in exon 14 of ATP7B gene. c.3121 is indicated by arrows. (D) c.3121C $>$ T mutation was inherited from the mother of the patient. (E) The father of the patient did not have the heterozygous mutation.

automated sequencer (Applied Biosystems; Thermo Fisher Scientific, Inc.).

Bioinformatics analysis. Bioinformatics analysis of the 2,000 bp sequence surrounding the two breakpoints using the RepeatMasker program 4.0.7 (http://www.repeatmasker.org/) showed special elements. The secondary structures of nucleotides for the 1,000 bp sequence in the vicinity of proximal and distal breakpoints were predicted using the UNAFold v3.8 suite (http://unafold.rna.albany.edu/?q=mfold/DNA-Folding-Form).

\section{Results}

The patient was a 2.7-year-old Chinese girl, born (weight, $3 \mathrm{~kg}$ ) to non-consanguineous parents as their only child following a pregnancy of normal duration. The patient presented with two-month history of anorexia, which was the only presenting symptom. The physical examination of the patient showed that her liver was palpable $\sim 4 \mathrm{~cm}$ under the low processus xiphoideus, and $\sim 3 \mathrm{~cm}$ under the right lowest rib. The texture was soft, the edge was blunt and there was no tenderness. The spleen was impalpable. KF rings were not observed. The patient had no neurological or psychological symptoms.

The clinical data collected from the patient on admission are presented in Table I. The laboratory tests identified a low level of serum CER, and high levels of ALT and AST. The MRI results of the brain were normal. The abdominal CT showed increased liver volume. The mean CT value of liver was $\sim 35$ HU (Hounsfield units), with spleen parenchymal CT values on the same plane of $\sim 50 \mathrm{HU}$. The decreased density of the liver had been proposed because the CT value of the liver was less than the spleen on basis of the literature report (7) (Fig. 1A and B). The parents of the patient were asymptomatic and so no laboratory tests, CT scans or MRI examinations were performed. Liver function of the patient was normal after treatment with penicillamine and zinc sulfate.

Mutation analysis of $A T P 7 B$ showed that the proband was compound heterozygous for c.3121C>T (p. Arg1041Trp) in exon 14, as assessed by Sanger sequencing (Fig. 1C) and had a large deletion of exons 3-21 detected via MLPA (Fig. 2A). The confirmed known missense mutation c.3121C $>\mathrm{T}$ in exon 14 causes a substitution from arginine to tryptophan at position 1041, and occurs in the ATP loop of the copper transporting $\mathrm{P}$ type ATPase (8). The mutation was inherited from the mother, who did not present any signs of WD (Fig. 1D). For the mutant site, her mother was heterozygous, whereas her father presented a wild-type allele (Fig. 1E). Suggesting that the patient presented a heterozygous and not a homozygous mutation. $A T P 7 B$ deletion/duplication detection using MLPA was therefore performed, and MLPA results showed that the proband and her father have a heterozygous gross deletion (Fig. 2). In order to accurately identify the deletions, NGS analysis was performed. A heterozygous deletion of exons 2-21 in the proband was found by NGS. This 
A

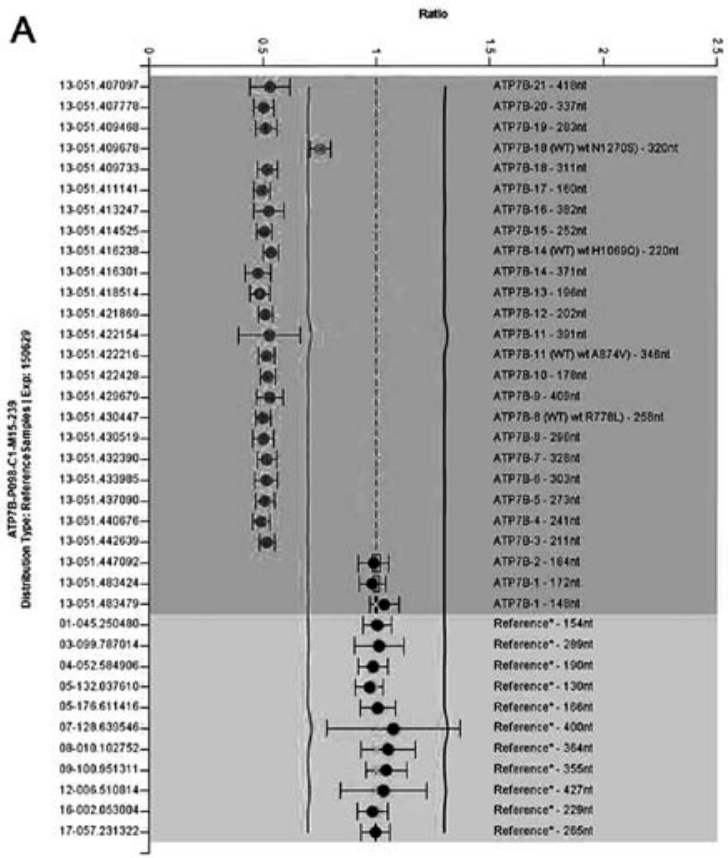

B

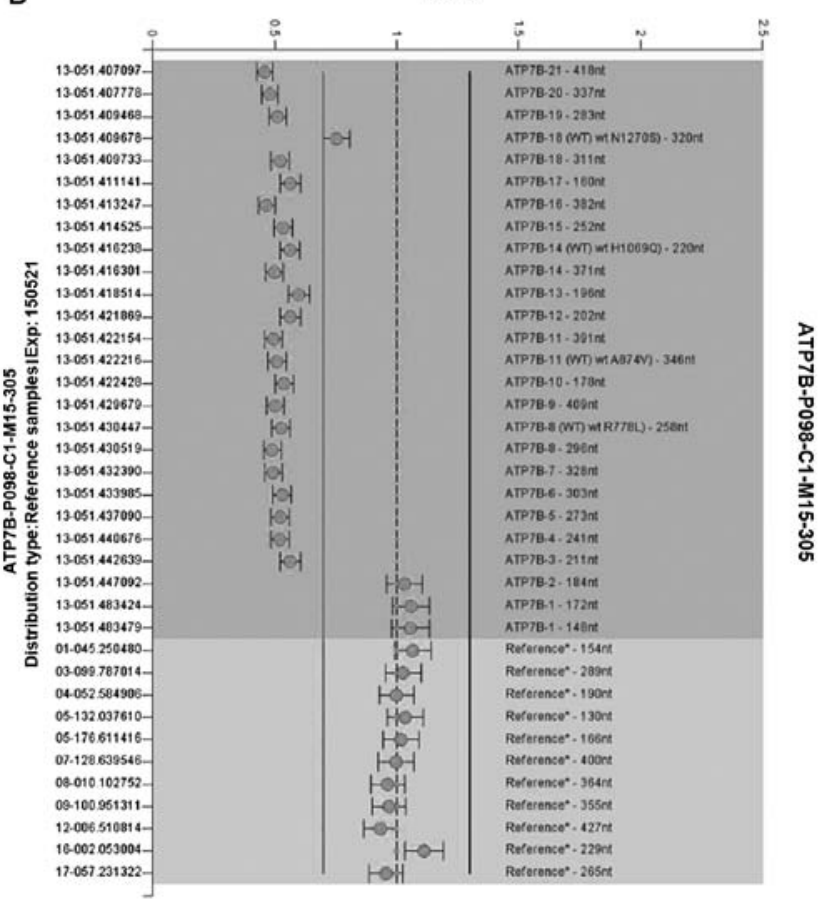

Figure 2. A heterozygous deletion analysis in the patient and her father using MLPA. (A) MLPA result of the patient with WD showing a heterozygous deletion of the exons 3-21 of the $A T P 7 B$ gene, as assessed by RPA ratios of 0.3-0.75. (Normal RPA ratio, 0.85-1.25 according to the manufacturer's recommendations). (B) The heterozygous gross deletion was inherited from the father of the patient. RPA, relative peak area; -represents the probe detection value.

mutation was identified as a novel gross deletion mutation in the $A T P 7 B$ gene sequence, which has never been previously reported. The large deletion was inherited from her father who was asymptomatic. There is a single copy exons $2-21$ of $A T P 7 B$ in the patient. The gross deletion mutation is predicted to cause the loss of almost all functional domains, including copper binding domains, transmembrane domains

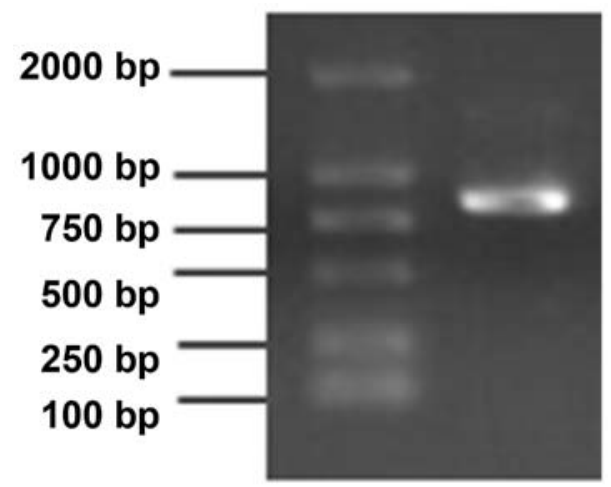

Figure 3. Putative breakpoint junction sequence was amplified by PCR. The $868 \mathrm{bp}$ band corresponding to the breakpoint junction sequence in the patient is presented in the second lane.

and the ATP loop (5). The present study found that the pathogenic mutations p.Arg1,041Trp and the gross deletion were compound heterozygous on two chromosomes in the proband, which were inherited separately from her mother and father. The sequencing analysis of the $868 \mathrm{bp}$ band of the amplified breakpoint junction showed a deletion of a $57,771 \mathrm{bp}$ fragment (chr13: 52490972-52548742)(GRCh37) from partial exons 2-21 to external $A T P 7 B$ sequence $(15.833 \mathrm{bp})$, without including additional genes (Fig. 3). The sequence of the breakpoint junction was confirmed in the proband and her father (Fig. 4A-C). The probe sequence (5'-GTCATGTGT GAAGTCCATTGAGGA-3') of MLPA for exon 2 of ATP7B binds within the remaining 562 bp of exon 2 (Fig. 4A), and for this reason MLPA failed to detect the deletion of exon 2 in the $A T P 7 B$ gene.

The sequence analysis of both sides of the breakpoints identified microhomology of one base $(\mathrm{G})$ next to inverted repeat sequences CATG-GTAC (Fig. 4A).

Bioinformatics analysis of the $2,000 \mathrm{bp}$ sequence surrounding the two breakpoints showed no consequential elements. The secondary structures of nucleotides for the $1,000 \mathrm{bp}$ sequence in the vicinity of proximal and distal breakpoints were predicted that the proximal breakpoint was found in the proximity of a hairpin structure (Fig. 4D and E).

The deletion may be caused by the inverted repeat sequences CATG-GTAC, which may lead to genomic instability, and by the microhomology sequence of one base $(\mathrm{G})$ at the proximal breakpoint, which may interact with the base $\mathrm{C}$ on the complementary strand in the proximity of the distal breakpoint, mediating non-homologous end joining repair. The same heterozygous deletion and breakpoint junction sequence were also present in the father of the proband (Fig. 4C), suggesting that the deletion event occurred in the family of the father of the proband.

\section{Discussion}

Wilson disease (WD) is a rare autosomal recessive genetic disorder that causes abnormal copper metabolism, which may result in a pathological accumulation of copper in the liver, brain and other organs (9). Diagnostic criteria are based on symptoms, biochemical testing and the presence of KF rings (10). Patients with cirrhosis, neurological manifestations 
A

ATCTTATCTAAGCTTTCTTTGCCTACCCGTGCCTGGGAACCAGCAATGAAGAAGAGTTTTGCTTTIGA CAATGTTGGCTATGAAGGTGGTCTGGATGGCCTGGGCCCTTCTTCTCAGGTGGCCACCAGCACAGTC AGGATCTTGGGCATGACTTGCCAGTCATGTGTGAAGTCCATTGAGGACAGGATTICCAATTTGAAA GCATCATCAGCATGAAGGTTICCCTGGAACAAGGCAGTGCCACTGTGAAATATGTGCCATCGGTTGT GTGCCTGCAACAGGTTIGCCATCAAATTGGGGACATGGGCTTCGAGGCCAGCATIGCAGAAGGAAA GGCAGCCTCCTGGCCCTCAAGGTCCTTGCCTGCCCAGGAGGCTGTGGTCAAGCTCCGGGTGGAGGGC ATGACCTGCCAGTCCTGTGTCAGCTCCATTGAAGGCAAGGTCCGGAAACTGCAAGGAGTAGTGAGAG TCAAAGTCTCACTCAGCAACCAAGAGGCCGTCATCACTTATCAGCCTTATCTCATTCAGCCCGAAGA CCTCAGGGACCATGTAAATGACATGGgattigaagegggtagaagGGTACTGAATTCTIGCTGGTTTAATTGAAGGGGGAAAGAGTG-

B C

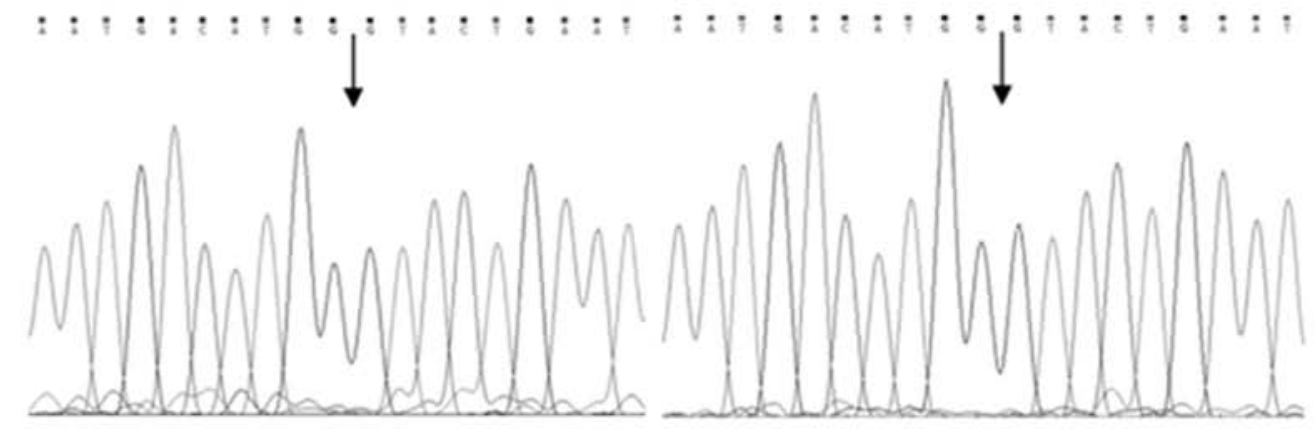

D

E
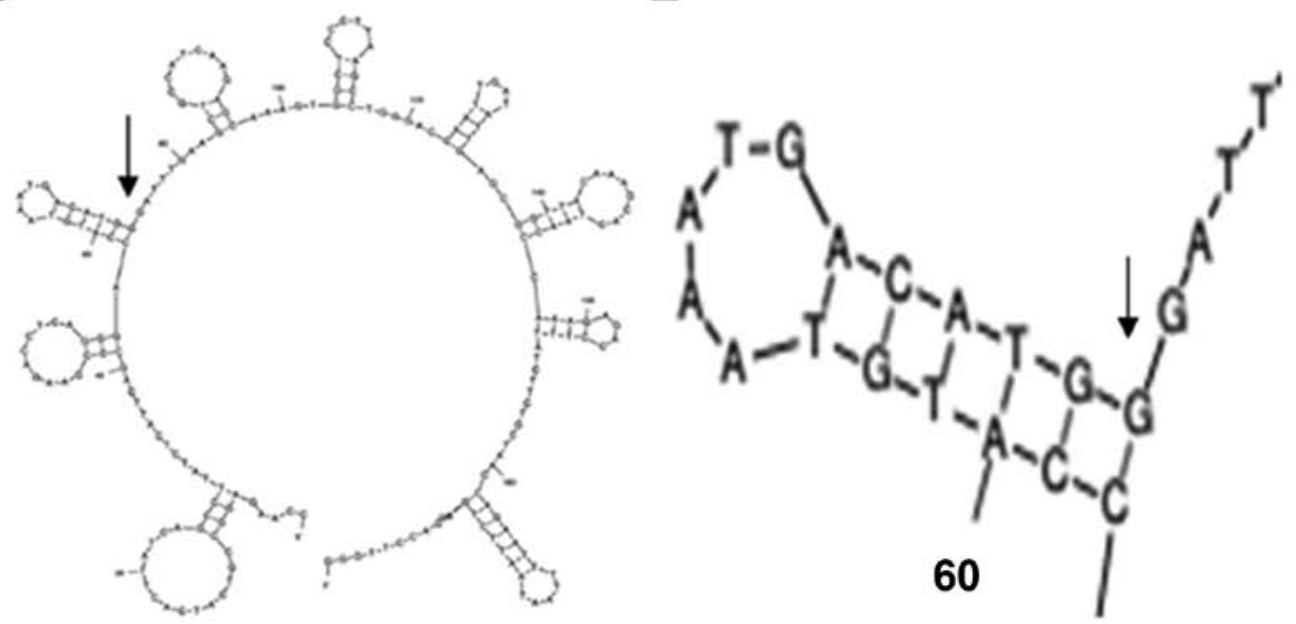

Figure 4. Analysis of the breakpoints. (A) Sequence characteristics around the breakpoints. Nucleotides underlined by a dotted line in exon 2 are targeted by the probe used for multiplex ligation-dependent probe amplification. Underlined nucleotides represent the Microhomology part. Bold nucleotides are inverted repeat sequences. Small case letters indicate the extremities of the deleted sequence. (B) Direct sequencing showed the breakpoint junction sequence in the patient. Breakpoint junction is indicated by an arrow. (C) The father presented the same breakpoint junction, indicated by an arrow. (D) DNA secondary structure in the vicinity of the proximal breakpoint was predicted using bioinformatic tools. The breakpoint was predicted to be in the proximity of a hairpin structure and is indicated by an arrow. (E) Magnification of the proximal breakpoint, which is indicated by an arrow.

and KF rings are diagnosed with classic WD. The type of clinical symptoms can be highly variable in pediatric patients with WD, as hepatic symptoms usually appear in late childhood and vary from asymptomatic, exhibiting only biochemical abnormalities, to acute, presenting liver failure and neurological or psychiatric manifestations that generally occur in the second or third decade of life (11-13). Laboratory diagnosis is based on low serum CER, increased 24-h urine copper excretion and elevated liver copper levels (10). The presence of $\mathrm{KF}$ rings reflects copper deposition in the brain (11). KF rings are usually absent in pediatric patients with WD with liver disease in the earlier period of the disease, and are not found in all patients with WD. KF rings are not specific to WD, and have also been seen in other forms of chronic liver diseases (14). When symptoms are mild in the early stages of WD, genetic investigation of $A T P 7 B$ mutations can be valuable in confirming a diagnosis of WD (11). Early diagnosis and treatment can effectively prevent excessive copper accumulation and tissue damage (15).

In the present study, a pediatric patient presented with early-onset hepatic disease. The patient did not present neurological or psychiatric manifestations, or KF rings. Laboratory 
tests showed a low level of serum CER, and high levels of ALT and AST. Biochemical investigations, including 24-h urine copper excretion and hepatic biopsy, were not carried out due to technical limitations. The early diagnosis of the patient with WD was made by testing for $A T P 7 B$ mutations. The early diagnosis of WD allowed a timely treatment.

Previous molecular genetic analyses identified $>1,000$ distinct mutations associated with WD worldwide, the most common mutations are the point mutations, and other type of mutations are rare (http://www.wilsondisease.med. ualberta.ca/database.asp). The distribution of mutations in the $A T P 7 B$ gene for WD are associated with ethnicity or geographic origin (11). The most prevalent mutation of the $A T P 7 B$ among Europeans and US Caucasian is p.H1069Q in exon $14(16,17)$, whereas the mutation p.R778L in exon 8 is most common in East Asia including China, South Korea and Japan (18-20).

The present study identified a previously described heterozygous mutation (p.Arg1,041Trp) and a heterozygous gross deletion including exons 2-21. The Arg1,041Trp mutation was first reported in an Italian patient in 1998 (8), and has never been reported previously in China, whereas, according to our knowledge, the heterozygous gross deletion including exons 2-21 of the $A T P 7 B$ gene has not been previously reported. In the present study, the mutations p.Arg1,041Trp and the gross deletion including exons 2-21 could alter the conformation of the ATP loop domain and all functional domains, respectively (5); thus resulting in a severe impairment of P-type ATPase function. The gross deletion mutation in the patient may be classified as a severe mutation, causing early hepatic disease onset. In the present study, the pediatric patient lacked the typical symptoms of WD and presented indeterminate biochemical results, which made early diagnosis more difficult. Therefore, molecular analysis of $A T P 7 B$ mutations may facilitate an early diagnosis of WD. A missense mutation was found in the homozygous state, due to the large heterozygous gene deletion on its corresponding allele, but no homologous mutations were inherited from the parents of the patient. Molecular diagnosis of WD in the patient was carried out using a combination of direct sequencing, MLPA and NGS. MLPA and NGS were used for the detection of exonic deletions/duplications of genomic DNA sequences. This analysis is of particular importance when $\leq 1$ mutation is detected in a patient with clinical symptoms of WD, as the limitations of traditional Sanger sequencing mean large deletions/duplications cannot be detected. MLPA can only detect exon deletions/duplications because of restricted location of probes. However, in the present study, NGS in combination with Sanger sequencing was more effective than MLPA in detecting precise deletions.

Microhomology sequences that are closely associated with deletion mutations in the vicinity of deletion breakpoints have previously been reported in the $A T P 7 B$ gene, including c.51 + 384_1708-953del (21), c.1870-45_2355+189del(22),c.4021+87_4125-2del(23),c.3556 +281_4001del, c.3134_3556 + 689del (24), c.532_574del (25), c.52-9329_1286-11del, c.2447 + 1612_2730 + 31delins12bp and c.3700-592_*409del (26). Alu repeat elements related to deletion events have also been discovered in the $A T P 7 B$ gene [c.3134_3556 + 689del (24) and c.52-2671_368del (27)]. In addition, sequence analysis surrounding the breakpoints of the deletion identified microhomology and inverted repeat sequences in the patient enrolled in the present study, which may also mediate deletion mutations in the $A T P 7 B$ gene.

The present study reported the clinical and molecular characterization of one Chinese pediatric patient with early onset WD, with liver disease as the presenting feature. In the patient, a heterozygous mutation (p. Arg1041Trp) and a heterozygous gross deletion of a 57,771 bp fragment (chr13: 52490972-52548742) from partial exons 2-21 to external ATP7B sequence $(15.833 \mathrm{bp})$ were identified through direct sequencing and NGS. Microhomology and inverted repeat sequences were found in the proband, which warrants further investigation of DNA break and recombination mechanisms. To the best of our knowledge, the Arg1041Trp mutation has not been previously reported in Chinese patients, and the novel heterozygous gross deletion mutation has not previously been identified, expanding the mutational spectrum of the $A T P 7 B$ gene. The present results suggested that the NGS assay in combination with Sanger sequencing of the breakpoint junctions may be more efficient than MLPA in detecting precise deletions.

\section{Acknowledgements}

The authors would like to thank Dr Haijian-Cheng (Beijing Kangso Medical Laboratory Co., Ltd., Beijing, China) for genetic data analysis.

\section{Funding}

The present work was supported by the Scientific Research Projects of Guizhou Health Commission in China (grant no. gzwjkj2018-1-048).

\section{Availability of data and materials}

The datasets used and/or analyzed during the current study are available from the corresponding author on reasonable request.

\section{Authors' contributions}

WLL, FL and LL carried out the genetic studies, the data analysis and wrote the manuscript. ZXH performed the genetic studies, the data analysis and manuscript revision. WC, HG and RA contributed to the clinical diagnosis and the clinical analysis. All authors read and approved the final manuscript.

\section{Ethics approval and consent to participate}

The study was approved by the Ethics Committees of Affiliated Hospital of Guizhou Medical University (approval no. 201011). Written informed consent was obtained from the parents of the patient.

\section{Patient consent for publication}

Not applicable.

\section{Competing interests}

The authors declare that they have no competing interests. 


\section{References}

1. Danks DM: Disorders of copper transport. In: The metabolic basis of inherited diseases. Scriver CR, Beaudet AL, Sly WS and Valle D (eds). Vol 1. 6th edition. McGraw-Hill, New York, NY, pp1416-1422, 1989.

2. Scheinberg IH and Sternlieb I: Wilson disease. In: Major Problems in Internal Medicine. Lloyd $\mathrm{H}$ and Smith J (eds). Saunders, Philadelphia, PA, 1984.

3. Sternlieb I: Perspectives on Wilson's disease. Hepatology 12: 1234-1239, 1990.

4. Petrukhin K, Fischer SG, Pirastu M, Tanzi RE, Chernov I, Devoto M, Brzustowicz LM, Cayanis E, Vitale E and Russo JJ: Mapping, cloning and genetic characterization of the region containing the Wilson disease gene. Nat Genet 5: 338-343, 1993

5. Terada K, Schilsky ML, Miura N and Sugiyama T: ATP7B (WND) protein. Int J Biochem Cell Biol 30: 1063-1067, 1998.

6. Thomas GR, Forbes JR, Roberts EA, Walshe JM and Cox DW: The Wilson disease gene: Spectrum of mutations and their consequences. Nat. Genet 9: 210-217, 1995.

7. Piekarski J, Goldberg HI, Royal SA, Axel L and Moss AA: Difference between liver and spleen CT numbers in the normal adult: Its usefulness in predicting the presence of diffuse liver disease. Radiology 137: 727-729, 1980.

8. Loudianos G, Dessì V, Lovicu M, Angius A, Nurchi A, Sturniolo GC, Marcellini M, Zancan L, Bragetti P, Akar N, et al: Further delineation of the molecular pathology of wilson disease in the mediterranean population. Hum Mutat 12: 89-94,1998.

9. Subramanian I, Vanek ZF and Bronstein JM: Diagnosis and treatment of Wilson's disease. Curr Neurol Neurosci Rep 2: 317-323, 2002.

10. Medici V, Rossaro L and Sturniolo GC: Wilson disease-A practical approach to diagnosis, treatment and follow-up. Dig Liver Dis 39: 601-609, 2007.

11. Das SK and Ray K: Wilson's disease: An update. Nat Clin Pract Neurol 2: 482-493, 2006.

12. Brewer GJ and Askari FK: Wilson's disease: Clinical management and therapy. J Hepatol 42 (Suppl): S13-S21, 2005.

13. Hedera P: Update on the clinical management of Wilson's disease. Appl Clin Genet 10: 9-19, 2017.

14. Mak CM and Lam CW: Diagnosis of Wilson's disease: A comprehensive review. Crit Rev Clin Lab Sci 45: 263-290, 2008.

15. Dalvi A and Padmanaban M: Wilson's disease: Etiology, diagnosis, and treatment. Dis Mon 60: 450-459, 2014.

16. Ferenci P: Regional distribution of mutations of the ATP7B gene in patients with Wilson disease: Impact on genetic testing. Hum Genet 120: 151-159, 2006.

17. Shah AB, Chernov I, Zhang HT, Ross BM, Das K, Lutsenko S, Parano E, Pavone L, Evgrafov O, Ivanova-Smolenskaya IA, et al: Identification and analysis of mutations in the Wilson disease gene (ATP7B): Population frequencies, genotype-phenotype correlation, and functional analyses. Am J Hum Genet 61: 317-328, 1997.
18. Liu XQ, Zhang YF, Liu TT, Hsiao KJ, Zhang JM, Gu XF, Bao KR, Yu LH and Wang MX: Correlation of ATP7B genotype with phenotype in Chinese patients with Wilson disease. World J Gastroenterol 10: 590-593, 2004.

19. Kim EK, Yoo OJ, Song KY, Yoo HW, Choi SY, Cho SW and Hahn SH: Identification of three novel mutations and a high frequency of the Arg778Leu mutation in Korean patients with Wilson disease. Hum Mutat 11: 275-278, 1998.

20. Okada T, Shiono Y, Hayashi H, Satoh H, Sawada T, Suzuki A, Takeda Y, Yano M, Michitaka K, Onji M and Mabuchi H: Mutational analysis of ATP7B and genotype-phenotype correlation in Japanese with Wilson's disease. Hum Mutat 15: 454-462, 2000.

21. Incollu S, Lepori MB, Zappu A, Dessì V, Noli MC, Mameli E, Iorio R, Rannuci G, Cao A and Loudianos G: DNA and RNA studies for molecular characterization of a gross deletion detected in homozygosity intheNH2-terminal region of the ATP7B gene in a Wilson disease patient. Mol Cell Probes 25: 195-198, 2011.

22. Tatsumi Y, Shinohara T, Imoto M, Wakusawa S, Yano M, Hayashi K, Hattori A, Hayashi H, Shimizu A, Ichiki T, et al: Potential of the international scoring system for the diagnosis of Wilson disease to differentiate Japanese patients who need anti-copper treatment. Hepatol Res 41: 887-896, 2011.

23. Møller LB, Ott P, Lund C and Horn N: Homozygosity for a gross partial gene deletion of the C-terminal end of ATP7B in a Wilson patient with hepatic and no neurological manifestations. Am J Med Genet A 138: 340-343, 2005.

24. Todorov T, Balakrishnan P, Savov A, Socha P and Schmidt HH: Intragenic deletions in ATP7B as an unusual molecular genetics mechanism of Wilson's disease pathogenesis. PLoS One 11: e0168372, 2016.

25. Liu G, Ma D, Cheng J, Zhang J, Luo C, Sun Y, Hu P, Wang Y, Jiang $\mathrm{T}$ and $\mathrm{Xu} \mathrm{Z}$ : Identification and characterization of a novel 43-bp deletion mutation of the ATP7B gene in a Chinese patient with Wilson's disease: A case report. BMC Med Genet 19: 61, 2018.

26. Chen YC, Yu H, Wang RM, Xie JJ, Ni W, Zhang Y, Dong Y and $\mathrm{Wu} \mathrm{ZY}$ : Contribution of intragenic deletions to mutation spectrum in Chinese patients with Wilson's disease and possible mechanism underlying ATP7B gross deletions. Parkinsonism Relat Disord 62: 128-133, 2019.

27. Mameli E, Lepori MB, Chiappe F, Ranucci G, Di Dato F, Iorio R and Loudianos G: Wilson's disease caused by alternative splicing and Alu exonization due to a homozygous 3039-bp deletion spanning from intron 1 to exon 2 of the ATP7B gene. Gene 569: 276-279, 2015

This work is licensed under a Creative Commons Attribution-NonCommercial-NoDerivatives 4.0 International (CC BY-NC-ND 4.0) License. 